\title{
On Multiplicities of Non-isolated Intersection Components
}

\author{
By \\ Wolfgang VoGeL*
}

\section{$\S 0$. Introduction}

In 1942, B.L. van der Waerden observed a very interesting fact in [24]. (We translate here from the original German): "However, to the best of my knowledge, the multiplicity of a non-isolated intersection point of 3 surfaces has never yet been defined." (For a wealth of background material see, e.g., $[9,10,13,21]$. Of course, it is very difficult to investigate embedded primary components, see, e.g., [11].)

The focus of this paper is a discussion of this observation. First, our theorem 1 shows that indeed we need contributions of non-isolated intersection components to the intersection theory in $\mathbb{P}^{n}$ as developed in [4] and [18, 20] (see [7,3] for the relation between [4] and [20].). Second, our theorem 2 establishes that non-isolated intersection points of 3 surfaces have not always multiplicities. Third, our theorem 3 shows that certain non-isolated intersection points do indeed have multiplicities. This is demonstrated by examples. Here is our first example (see also [19]).

Example. Let $X$ and $Y$ be subschemes of $\boldsymbol{P}^{3}$ with defining ideals

$$
I(X)=\left(X_{1}^{2}, X_{0} \cdot\left(X_{1} X_{3}-X_{2}^{2}\right)\right) \text { and } I(Y)=\left(X_{2}^{2}\right) .
$$

We obtain the following primary decomposition:

$$
I(X)+I(Y)=\left(X_{1}, X_{2}^{2}\right) \cap\left(X_{0}, X_{1}^{2}, X_{2}^{2}\right) \cap\left(X_{1}^{2}, X_{2}^{2}, X_{3}\right) .
$$

Then the line given by $X_{1}=X_{2}=0$ and the non-isolated intersection point defined by $X_{0}=X_{1}=X_{2}=0$ are counted with multiplicities 8 and 4 , resp. Moreo-

Communicated by K. Saito, September 27, 1990.

1991 Mathematics Subject Classification: 14C17, 13H15

* Martin-Luther-University, Dept. of Mathematics, O-4010 Halle, Germany. 
ver, the embedded intersection point given by $X_{1}=X_{2}=X_{3}=0$ has no multiplicity in the sense of the intersection theory as presented in $[4,20]$.

Therefore the main purpose of this paper is to describe contributions of non-isolated components to the intersection theory. Hence our approach also yields new results concerning a converse of Bézout's theorem (see [2,3, $15,21,22])$. Finally, we conclude by studying some examples and problems.

\section{§1. Notations and Prelinminary Results}

Before embarking on the proofs of the theorems we must prove several preliminary results. First we want to recall the main theorem of $[18,20]$. Let $X, Y$ be pure dimensional subschemes of $\mathbb{P}_{k}^{n}$ with defining ideals $\mathbb{I}(X)$ and $\mathbb{I}(Y)$ in $k\left[x_{0}, \cdots, x_{n}\right]=: \mathbb{R}_{x}$ over a field $k$. We introduce a second copy $k\left[y_{0}, \cdots, y_{n}\right]=: \mathbb{R}_{y}$ of $\mathbb{R}_{x}$ and denote by $\mathbb{I}(Y)^{\prime}$ the ideal in $\mathbb{R}_{y}$ corresponding to $I(Y)$. Furthermore, we introduce new independent variables $u_{i, m}$ over $k$ for $i, m=0,1, \cdots, n$. Let $K$ be the field extension $k\left(u_{00}, \cdots, u_{n n}\right)$. We consider the polynomial ring $R=K\left[x_{0}, \cdots, x_{n}, y_{0}, \cdots, y_{n}\right]$, and forms of degree 1 in $R$ :

$$
l_{i}=\sum_{m=0}^{n} u_{i m}\left(x_{m}-y_{m}\right) \quad \text { for } \quad i=0, \cdots, n \text {. }
$$

We note that $\left(l_{0}, \cdots, l_{n}\right) \circ R=\left(x_{0}-y_{0}, \cdots, x_{n}-y_{n}\right) \cdot R=: c$. We set

$$
\begin{aligned}
& \delta:=\mathbb{K} \text { rull-dimension of } \mathbb{I}(X)+\mathbb{I}(Y)^{\prime} \text { in } R, \\
& d:=\operatorname{Krull}-\operatorname{dimension} \text { of } \mathbb{I}(X)+\mathbb{I}(Y) \text { in } \mathbb{R}_{x}, \text { and } \\
& e:=\operatorname{dim} X \cap Y-(\operatorname{dim} X+\operatorname{dim} Y-n), \text { that is, }
\end{aligned}
$$

$e$ is the excess dimension of $X$ and $Y$.

We note that $n-e=\delta-d-1$.

Take the linear forms $l_{0}, \cdots, l_{\delta-d-1}$ and put $\left(I(X) \cdot R+I(Y)^{\prime} R\right)_{-1}:=\mathbb{I}(X) R+$ $I(Y)^{\prime} R$, and

$$
\left(I(X) R+\mathbb{I}(Y)^{\prime} R\right)_{m}:=l_{m} \circ \mathbb{R}+U\left(\left(I(X) \mathbb{R}+\mathbb{I}(Y)^{\prime} \mathbb{R}\right)_{m-1}\right)
$$

for any $m=0, \cdots, \delta-d-1$, where $U(\cdots)$ is the intersection of all highest dimensional primary ideals belonging to the ideal $(\cdots)$.

Furthermore, we put

$$
a_{0}:=U\left(\left(I(X) R+\mathbb{I}(Y)^{\prime} R\right)_{\delta-d-2}\right. \text {, and }
$$

$a_{s}:=$ intersection of all primary ideals belonging to $U\left(\mathfrak{a}_{s-1}+l_{\delta-d+s-2} R\right)$ such that $\mathfrak{c} R$ is not contained in their associated primes for all $s=1, \cdots$, $e+1$ provided $\mathfrak{a}_{s-1} \neq R$. 
Following $[18,20,2]$ we want to recall the construction of the collection $\mathcal{C}$ and the definition of the intersection multiplicities $j(X, Y ; C)$ and $k(X, Y ; C)$ for all elements $C$ of $\mathcal{C}$.

The collection $\mathcal{C}$ contains irreducible and reduced subvarieties, say $C$, of $X \cap Y$ lying in $\mathbb{P}_{K}^{n}$ with the property that $\operatorname{dim} X \cap Y \geqq \operatorname{dim} C \geqq \operatorname{dim} X+$ $\operatorname{dim} Y-n$. We shall denote here this collection of subvarieties by $\mathcal{C}(X, Y)$. When there is no possibility of confusion we will denote $\mathcal{C}(X, Y)$ simply by $\mathcal{C}$.

The elements of $\mathcal{C}$ of dimension $\operatorname{dim} X \cap Y-i$ for $i=0, \cdots, e$ are defined as follows:

Consider all prime ideals belonging to $U\left(\mathfrak{a}_{i}+l_{n-e+i} R\right)$ and containing the ideal $c \cdot R$. Then these prime ideals modulo $c \cdot R$ define irreducible and reduced subvarieties of $X \cap Y$ in $\mathbb{P}_{K}^{n}$. These subvarieties are the elements of $\mathcal{C}$ of dimension equal to $\operatorname{dim} X \cap Y-i$.

Moreover, we would like to recall the definition of the intersection multiplicities $j(X, Y ; C)$ and $k(X, Y ; C)$ of $X$ and $Y$ along $C$ for all $C \in \mathcal{C}$. Let $C$ be an element of $\mathcal{C}$ of dimension equal to $\operatorname{dim} X \cap Y-i$. Let $I(C)$ be the defining prime ideal of $C$ in $K\left[x_{0}, \cdots, x_{n}\right]$. We then set

$$
j(X, Y ; C):=\text { length of } U\left(\mathfrak{a}_{i}+l_{n-e+i} R\right)_{I(C) R+c \cdot R}
$$

and

$$
\begin{aligned}
k(X, Y ; C): & =\text { length of }\left(U\left(\mathfrak{a}_{i}+l_{n-e+i} R\right)+\mathfrak{c} \cdot R\right)_{I(C) R+c \cdot R} \\
& \leqq j(X, Y ; C)
\end{aligned}
$$

Hence both intersection numbers are defined by the length of well-defined primary ideals of the polynomial ring $K\left[x_{0}, \cdots, x_{n}, y_{0}, \cdots, y_{n}\right]$. Hence the above definitions describe an intersection algorithm in the ruled join construction of $\mathbb{P}_{K}^{2 n+1}$ (for a wealth of background material on this join construction, see, e.g., $[5,8])$.

It follows immediately from the above construction that every irreducible component of $X \cap Y$ belongs to $\mathcal{C}$. We denote by $\mathcal{C}_{\mathrm{irr}}$ the collection of irreducible components of $X \cap Y$. We set

$$
\mathcal{C}_{h}:=\{C \in \mathcal{C} \text { with } \operatorname{dim} C=\operatorname{dim} X \cap Y\} .
$$

Observe that

$$
\mathcal{C}_{h} \subseteq \mathcal{C}_{\mathrm{irr}} \subseteq \mathcal{C}
$$

But we also have elements of $\mathcal{C}$ defined by certain embedded prime ideals belonging to $I(X)+I(Y)$ (see, e.g., our example of the introduction). More- 
over, our algorithm also does yield some extra components of $X \cap Y$ defined over $k$ or $K$ (see Example 2 of $\S 4$ ). Therefore an important object is the (dim $X \cap Y-i$ )-dimensional cycle

$$
V_{i}=\sum j(X, Y ; C) C,
$$

where the sum is taken over all $C \in \mathcal{C}$ of dimension equal to $\operatorname{dim} X \cap Y-i$ for $i=0, \cdots, e$. This shows that the Segre-Mather classes of [25] naturally appears in our cycle theory (see also $[7,3]$ ). Hence our above intersection algorithm does yield an affirmative answer to some questions asked in [26], (2.6) and (3.5).

We need main results of $[18,20]$ and $[2,3]$.

Lemma 1 (Refined Bézout's theorem of $[18,20]$ ). Let $X$ and $Y$ be pure dimensional subschemes of $\mathbb{P}_{k}^{n}$. Then we have

$$
\text { degree } X \text {. degree } Y=\sum_{C \in C} j(X, Y ; C) \text { degree } C
$$

Lemma 2 [(2)]. Let $X$ and $Y$ be reduced and pure dimensional subschemes of $\mathbb{P}_{k}^{n}$. Moreover, we assume that $X$ and $Y$ are connected in dimension $\operatorname{dim} X-1$ and $\operatorname{dim} Y-1$, resp. Let $X \cup Y$ be not lying in a hyperplane. Then we have

$$
\begin{aligned}
& \text { degree } X \cdot \text { degree } Y \geqq \sum_{C \in C_{\mathrm{irr}}} k(X, Y ; C) \text { degree } C+e, \\
& \text { where } e=\operatorname{dim} X \cap Y-(\operatorname{dim} X+\operatorname{dim} Y-n) .
\end{aligned}
$$

As a corollary we therefore obtain a converse of Bézout's theorem. $e=0$.

Corollary. degree $X \cdot$ degree $Y \leqq \sum_{C \in C_{\mathrm{irr}}} k(X, Y ; C)$ degree $C$ if and only if

We will also apply a certain bilinear property of the above intersection algorithm in the join construction of $\mathbb{P}_{K}^{2 n+1}$. We therefore state a theorem of additivity and a reduction theorem needed for the proof of our theorems. There are different arguments in proving this result. For example, L. van Gastel $[6,7]$ described geometrically and partly generalized the intersection theory of $[18,20]$. Hence the following lemma is clear from the definition of the above algorithm (see [6], Remark 4.4).

Lemma 3. Let $X, Y$ be pure dimensional subschemes of $\mathbb{P}_{k}^{n}$ with defining ideals $I(X)$ and $I(Y)$ in $K\left[x_{0}, \cdots, x_{n}\right]$.

We consider primary decompositions of $I(X)$ and $I(Y)$, say $I(X)=\mathrm{q}_{1} \cap \cdots \cap \mathfrak{q}_{r}$ where $\mathfrak{q}_{i}$ is $\mathfrak{p}_{i}$-primary, and $I(Y)=\mathfrak{q}_{1}^{\prime} \cap \cdots \cap \mathfrak{q}_{s}^{\prime}$ where $\mathfrak{q}_{j}^{\prime}$ is $\mathfrak{p}_{j}^{\prime}$-primary. We set $X=\bigcup_{i=1}^{r} X_{i}$ and $Y=\bigcap_{j=1}^{s} Y_{j}$ where $X_{i}$ is defined by $\mathfrak{q}_{i}$, and $Y_{j}$ is given by $\mathfrak{q}_{j}^{\prime}$ for 
$i=1, \cdots, r$, and $j=1, \cdots, s$, resp. We define reduced and irreducible subschemes $V_{i}$ and $W_{j}$ defined by the prime ideals $\mathfrak{p}_{i}, 1 \leqq i \leqq r$, and $\mathfrak{p}_{j}^{\prime}, 1 \leqq j \leqq s$, resp., that is, $\left(X_{i}\right)_{\mathrm{red}}:=V_{i}$ and $\left(Y_{j}\right)_{\mathrm{red}}:=W_{j}$. We set

$$
\begin{aligned}
& l_{i}:=\text { length of } \mathfrak{q}_{i} \text { for } i=1, \cdots, r, \text { and } \\
& m_{j}:=\text { length of } \mathfrak{q}_{j}^{\prime} \text { for } j=1, \cdots, s .
\end{aligned}
$$

Then we have

(i) $\mathcal{C}(X, Y)=\bigcup_{i=1}^{r} \bigcup_{j=1}^{s} \mathcal{C}\left(V_{i}, W_{j}\right)$.

(ii) For every $C \in \mathcal{C}(X, Y)$ we get

$$
j(X, Y ; C)=\sum_{i=1}^{r} \sum_{j=1}^{s} l_{i} \cdot m_{j} \cdot j\left(V_{i}, W_{j} ; C\right),
$$

where we set $j\left(V_{i}, W_{j} ; C\right)=0$ if $C \notin \mathcal{C}\left(V_{i}, W_{j}\right)$.

Remark. Examples show that we cannot replace the intersection number $j(X, Y ; C)$ in (ii) of Lemma 3 by the intersection multiplicity $k(X, Y ; C)$. Analyzing such examples one might be tempted to ask the following question:

Let $X$ and $Y$ be irreducible subschemes of $\boldsymbol{P}_{k}^{n}$. Let $C$ be an irreducible component of $X \cap Y$. Is it true that

$$
k(X, Y ; C) \leqq \text { length } \mathcal{O}_{X, X_{\text {red }}} \cdot \text { length } \mathcal{O}_{Y, Y_{\text {red }}} \cdot k\left(X_{\text {red }}, Y_{\text {red }} ; C\right) ?
$$

However, this is not so, as we will show by Example 1 of $\S 4$. The construction of this example is based on joint discussions with H. Flenner (Göttingen).

\section{§ 2. Main Results}

Theorem 1. Let $X, Y$ be pure dimensional projective subschemes of $\boldsymbol{P}_{k}^{n}$, say $X=\bigcup_{i=1}^{r} X_{i}$ and $Y=\bigcup_{j=1}^{s} Y_{j}$ where $X_{i}$ and $Y_{j}$ are irreducible, and $\operatorname{dim} X=\operatorname{dim} X_{i}$, $\operatorname{dim} Y=\operatorname{dim} Y_{j}$ for all $i=1, \cdots, r$ and $j=1, \cdots, s . \quad$ Let $r_{i j} \geqq 0$ be the integer such that the reduced scheme $\left(X_{i} \cup Y_{j}\right)_{\mathrm{red}}$ is contained in an $\left(n-r_{i j}\right)$-plane but not lying in an $\left(n-r_{i j}-1\right)$-plane. Let $e \geqq 0$ be the excess dimension of $X$ and $Y$, that is, $e=\operatorname{dim} X \cap Y-(\operatorname{dim} X+\operatorname{dim} Y-n)$. Consider the following conditions:

(i) $e \leqq r_{i j}$ for all $i=1, \cdots, r$ and $j=1, \cdots, s$

(ii) $\operatorname{deg} X \cdot \operatorname{deg} Y=\sum_{C \in \mathcal{C}_{b}} j(X, Y ; C) \operatorname{deg} C$

(iii) $\operatorname{dim} X \cap Y=\operatorname{dim} X_{i} \cap Y_{j}$ for all $i=1, \cdots, r$ and $j=1, \cdots, s$.

Then we have

(a) (i) $\Rightarrow$ (ii) $\Rightarrow$ (iii)

(b) The implications (iii) $\Rightarrow$ (ii), and (ii) $\Rightarrow$ (i) are not true in general. 
Proof. (a): (i) $\Rightarrow$ (ii): We will show that the collection $\mathcal{C}(X, Y)=$ $C_{h}(X, Y)$. Therefore we consider the intersection algorithm in the join construction as developed in $[18,20]$ (see also $\S 1$ ) by taking always the radical of the corresponding ideals. Let $I\left(X_{\dot{\xi}}\right)$ and $I\left(Y_{j}\right)$ be the defining ideals of $X_{\dot{\varepsilon}}$ and $Y_{j}$, resp. for all $i=1, \cdots, r$ and $j=1, \cdots, s$.

Hence we get

$$
\operatorname{Rad}\left(I(X), \mathbb{I}(Y)^{\prime}, l_{0}, \cdots, l_{n-e}\right)=\bigcap_{i, j} \operatorname{Rad}\left(I\left(X_{i}\right), I\left(Y_{j}\right)^{\prime}, l_{0}, \cdots, l_{n-e}\right)
$$

The ideal $\operatorname{Rad} I\left(X_{i}\right) \cap \operatorname{Rad} I\left(Y_{j}\right)$ does contain $r_{i j}$ linear independent forms of degree 1. Since $r_{i j} \geqq e$ we therefore may assume that the $e$ elements $x_{n-e+1}$ $y_{n-e+1}, \cdots, x_{n}-y_{n} \in \operatorname{Rad}\left(I\left(X_{j}\right), I\left(Y_{j}\right)^{\prime}, l_{0}, \cdots, l_{n-e}\right)$. This provides that the ideal

$$
\mathfrak{c}=\left(x_{0}-y_{0}, \cdots, x_{n}-y_{n}\right) \subseteq \mathbb{R a d}\left(\mathbb{I}(X), \mathbb{I}(Y)^{\prime}, l_{0}, \cdots, l_{n-e}\right) .
$$

Therefore the intersection algorithm does not yield an ideal $a_{1}$. Hence the algorithm stops, that is, $\mathcal{C}=\mathcal{C}_{h}$. This shows our condition (ii). (ii) $\Rightarrow$ (iii) follows from the theorem of additivity and reduction (see Lemma 3 of $\S 1$ ).

It is easily to construct examples which show (b).

This completes the proof of Theorem 1, q.e.d.

Corollary 1.1. With the same notations as in the theorem, assume that all irreducible components of $X \cap Y$ have the same dimension. Suppose that there are integers $i, 1 \leqq i \leqq r$, and $j, 1 \leqq j \leqq s$ such that $\operatorname{dim} X \cap Y \neq \operatorname{dim} X_{i} \cap Y_{j}$ then there is at least one non-isolated component of $X \cap Y$ belonging to $\mathcal{C}(X, Y)$; that is, non-isolated components do yield contributions to the intersection theory.

Corollary $\mathbb{1}_{0}$. With the same notations as in the theorem, assume that there is an irreducible component of $X \cap Y$ with dimension $<\operatorname{dim} X \cap Y$. Then there are integers $i, 1 \leqq i \leqq r$, and $j, 1 \leqq j \leqq s$ such that $r_{i j}<e$.

Remark 1.3. We want to study our example from the introduction. We have $X=X_{1} \cup X_{2}$ with defining ideals $I\left(X_{1}\right)=\left(X_{1}^{2}, X_{1} X_{3}-X_{2}^{2}\right)$ and $I\left(X_{2}\right)=\left(X_{1}^{2}, X_{0}\right)$, and $Y=Y_{1}$ with $\mathbb{I}\left(Y_{1}\right)=\left(X_{2}^{2}\right)$. Hence $0=\operatorname{dim} X_{2} \cap Y_{1} \neq \operatorname{dim} X \cap Y=1$. Our corollary 1.1 therefore shows that non-isolated intersection points of $X \cap Y$ have multiplicities.

Theorem 2。 With the same notations as in Theorem 1, take any subvariety $C$ of $X \cap Y$ with $\operatorname{dim} X \cap Y>\operatorname{dim} C \geqq \operatorname{dim} X+\operatorname{dim} Y-n$. Set $c:=\operatorname{dim} X \cap Y-$ $\operatorname{dim} C \geqq 1$. Consider all integers $i, 1 \leqq i \leqq r$, and $j, 1 \leqq j \leqq s$, such that $C \leqq$ 
$X_{i} \cap Y_{j}$. We assume that $r_{i j} \geqq e-c+1$ for all these integers $i$ and $j$. Then $C$ does not belong to the collection $\mathcal{C}(X, Y)$, that is, $C$ does not yield a contribution to the intersection theory.

Proof. We consider again the intersection algorithm by taking always the radical of the corresponding ideals. Moreover, we regard this algorithm with respect to the localization at the prime ideal $I(C)+c$ where $I(C)$ is the defining ideal of $C$. Then we get

$$
\begin{aligned}
& \operatorname{Rad}\left(I(X)+I(Y)^{\prime}+\left(l_{0}, \cdots, l_{n-e}, l_{n-e+1}, \cdots, l_{n-e+c-1}\right)\right)_{I(c)+c} \\
= & \bigcap_{i, j} \operatorname{Rad}\left(\operatorname{Rad}\left(I\left(X_{i}\right)+I\left(Y_{j}\right)^{\prime}\right)+\left(l_{0}, \cdots, l_{n-e+c-1}\right)\right)_{I(c)+c} \\
\subseteq & \left(\operatorname{Rad}\left(\mathfrak{a}_{c}\right)\right)_{I(\mathcal{c})+\mathfrak{c} .}
\end{aligned}
$$

Since $r_{i j} \geqq e-c+1$ we obtain that

$$
\mathfrak{c} \cong \bigcap_{i, j} \operatorname{Rad}\left(\operatorname{Rad}\left(I\left(X_{i}\right)+I\left(Y_{j}\right)^{\prime}+\left(l_{0}, \cdots, l_{n-e+c-1}\right)\right)_{I(C)+} \cdot\right.
$$

Therefore we have $\mathfrak{c} \subseteq\left(\operatorname{Rad}\left(\mathfrak{a}_{c}\right)\right)_{I(D)+\mathfrak{c}}$. Hence $\left(\operatorname{Rad}\left(\mathfrak{a}_{c}\right)\right)_{I(\mathcal{c})+\mathfrak{c}}$ is the whole ring since the prime ideals belonging to $a_{c}$ do not contain the ideal $c$ (see the intersection algorithm of $\S 1$ ). Therefore the intersection algorithm shows that $C \notin C$, q.e.d.

Remark 2.1. We would like to study again our example from the introduction by applying Theorem 2 . With the same notations as in the introduction and Remark 1.3, consider the non-isolated intersection point $C$ defined by $x_{1}=x_{2}=x_{3}=0$. Then we get $c=1$ and $e=1$. Since $C \subseteq X_{1} \cap Y_{1}$ but $C \nsubseteq X_{2} \cap Y_{1}$ we only have $r_{11}=1$ satisfying our assumption of Theorem 2: $r_{11} \geqq e-c+1$. Therefore we obtain $C \notin \mathcal{C}$, that is, $C$ has no multiplicity in the sense of the intersection theory of $[4,20]$.

Theorem 3. With the same notations as in theorem 1, consider all irreducible intersection components of $X_{i} \cap Y_{j}$ for all $i=1, \cdots, r$ and $j=1, \cdots, s$. Then these components do belong to the collection $\mathcal{C}(X, Y)$, that is, such components do yield a contribution to the intersection theory.

Proof. Lemma 1 and Lemma 3 of $\S 1$.

Remark 3.1. Consider again our example from the introduction. With the same notations as in the introduction and Remark 1.3, consider the nonisolated intersection point $P$ defined by $x_{0}=x_{1}=x_{2}=0$. Then we see that $P$ is an isolated intersection point of $X_{2} \cap Y_{1}$. Therefore Theorem 3 shows that 
$P \in C(X, Y)$, that is, $P$ has a multiplicity in the sense of the intersection theory of $[4,20]$.

\section{§. On a Converse of Bézout's Theorem}

An aim of this section is to discuss the following problem (see [22], problem 1): Let $X, Y$ be pure dimensional subschemes of $\mathbb{P}^{n}$. Under which circumstances is the following implication true:

$$
\operatorname{deg} X \cdot \operatorname{deg} Y=\sum_{C \in \mathcal{C}_{\mathrm{irr}}} j(X, Y ; C) \cdot \operatorname{deg} C \Rightarrow e=0 \text { ? }
$$

Of course, examples show that this is not true in general (see, e.g., $[2,3,7]$ ). However, the following theorem yields some applications for a solution of this problem.

Theorem 4 . With the same notations as in Theorem 1, consider the following conditions:

(i) $\operatorname{deg} X \cdot \operatorname{deg} Y \leqq \sum_{C \in C_{\mathrm{irr}}} k(X, Y ; C) \cdot \operatorname{deg} C$

(ii) $r_{i j}=e_{i j}$ for all $i=1, \cdots, r$, and $j=1, \cdots, s$.

(iii) $\mathcal{C}(X, Y)=\bigcup_{i, j} \mathcal{C}_{h}\left(X_{i}, Y_{j}\right)$ for $i=1, \cdots, r$ and $j=1, \cdots, s$.

Then we have

(a) (i) $\Rightarrow$ (ii) $\Rightarrow$ (iii)

(b) The implications (iii) $\Rightarrow$ (ii), and (ii) $\Rightarrow$ (i) are not true in general.

Proof. (a): We will reach the desired result by a suitable deepening of the approach as presented in the proof of the theorem of [22] and [14].

(i) $\Rightarrow$ (ii). Consider the excess dimensions $e\left(X_{i}, Y_{j}\right)$ and $e\left(V_{i}, W_{j}\right)$, where $V_{i}$ and $W_{j}$ are the embeddings of the reduced schemes $\left(X_{i}\right)_{\mathrm{red}}$ and $\left(Y_{j}\right)_{\mathrm{red}}$, resp. in $\mathbb{P}^{n-r_{i j}}$. Then we have $e\left(X_{\dot{j}}, Y_{j}\right)=e\left(V_{i}, W_{j}\right)+r_{i j}$, that is, we have to show that $e\left(V_{i}, W_{j}\right)=0$ under the assumption (i). First, condition (i) gives:

$$
\begin{aligned}
\operatorname{deg} X \cdot \operatorname{deg} Y & \leqq \sum_{C \in \mathcal{C}_{\mathrm{irr}}} k(X, Y ; C) \operatorname{deg} C \\
& \leqq \sum_{C \in \mathcal{C}_{\mathrm{irr}}} j(X, Y ; C) \operatorname{deg} C \\
& \leqq \sum_{C \in C} j(X, Y ; C) \operatorname{deg} C=\operatorname{deg} X \operatorname{deg} Y
\end{aligned}
$$

Hence we obtain that $\mathcal{C}_{\text {irr }}(X, Y)=\mathcal{C}(X, Y)$, and also $j(X, Y ; C)=k(X, Y ; C)$ for all $C \in \mathcal{C}(X, Y)$. Therefore we get from Lemma 3 of $\S 1$ and Lemma 4 of [22] that $\mathcal{C}\left(V_{i}, W_{j}\right)=\mathcal{C}_{\mathrm{irr}}\left(V_{i}, W_{j}\right)$ and $j\left(V_{i}, W_{j} ; C\right)=k\left(V_{i}, W_{j} ; C\right)$ for all 
$C \in \mathcal{C}\left(V_{i}, W_{j}\right)$. These properties do yield the following:

$$
\operatorname{deg} V_{i} \cdot \operatorname{deg} W_{j} \geqq \sum_{C \in \mathcal{C}_{\mathrm{irr}}\left(V_{i}, W_{j}\right)} k\left(V_{i}, W_{j} ; C\right) \cdot \operatorname{deg} C+e\left(V_{i}, W_{j}\right)
$$

by Lemma 2 of $\S 1$

$$
\begin{aligned}
& \geqq \sum_{C \in C_{\mathrm{irr}}\left(V_{i}, W_{j}\right)} k\left(V_{i}, W_{j} ; C\right) \cdot \operatorname{deg} C \\
& =\sum_{C \in C\left(V_{i}, W_{j}\right)} j\left(V_{i}, W_{j} ; C\right) \cdot \operatorname{deg} C \\
& =\operatorname{deg} V_{i} \cdot \operatorname{deg} W_{j}
\end{aligned}
$$

by Lemma 1 of $\S 1$.

Therefore we have $e\left(V_{i}, W_{j}\right)=0$.

(ii) $\Rightarrow$ (iii): Condition (ii) provides that $e\left(V_{i}, W_{j}\right)=0$ for all $i=1, \cdots, r$ and $j=1, \cdots, s$; that is, $V_{i} \cap W_{j}$ is a proper intersection in $\mathbb{P}^{n-r_{i j}}$. Hence we have that $\mathcal{C}_{h}\left(V_{i}, W_{j}\right)=\mathcal{C}\left(V_{i}, W_{j}\right)$. There is a 1-1 correspondence between the elements of $\mathcal{C}\left(X_{i}, Y_{j}\right)=\mathcal{C}\left(\left(X_{i}\right)_{\mathrm{red}},\left(Y_{j}\right)_{\mathrm{red}}\right)$ and $\mathcal{C}\left(V_{i}, W_{j}\right)$. Therefore we obtain $C\left(X_{\boldsymbol{i}}, Y_{\boldsymbol{j}}\right)=\mathcal{C}_{h}\left(X_{\boldsymbol{i}}, Y_{\boldsymbol{j}}\right)$. Our lemma 3 yields condition (iii).

(b): Example 2 of [22] shows that the implication (iii) $\Rightarrow$ (ii) is not true in general.

(ii) $\Rightarrow$ (i): In $\mathbb{P}^{2}$ consider the curves $X$ and $Y$ with defining ideals $I(X)=$ $\left(X_{1} X_{2}\right)=\left(X_{1}\right) \cap\left(X_{2}\right)$ and $I(Y)=\left(X_{1}\right)$, resp. Hence we have $r_{11}=e_{11}=1$, and $r_{21}=$ $e_{21}=0$. But $2=\operatorname{deg} X \cdot \operatorname{deg} Y \neq k(X, Y ; C) \operatorname{deg} C$ where $C$ has defining ideal $I(C)=\left(X_{1}\right), k(X, Y ; C)=$ length $\mathcal{O}_{X \cap Y ; C}=1$ and $\mathcal{C}_{\mathrm{irr}}(X, Y)=\{C\}$. This completes the proof of Theorem 4, q.e.d.

Corollary 4.1. Let $X, Y$ be irreducible subschemes of $\mathbb{P}^{n}$ such that $(X \cup Y)_{\mathrm{red}} \subseteq \mathbb{P}^{n-r}$ but not in $\mathbb{P}^{n-r-1}$ with $r \geqq 0$. Assume that $e(X, Y)=r$ then we get

$$
\operatorname{deg} X \cdot \operatorname{deg} Y=\sum_{C \in \mathcal{C}_{h}(X, Y)} j(X, Y ; C) \operatorname{deg} C .
$$

Proof. Apply the implication (ii) $\Rightarrow$ (iii) of Theorem 4, q.e.d.

In the following we want to discuss the converse of Corollary 4.1. We need the following

Lemma 4. Let $X, Y$ be pure dimensional projective subschemes of $\mathbb{P}^{n}$. Let $C$ be an irreducible component of $X \cap Y$. We assume that the local rings $\mathcal{O}_{X, C}$ and $\mathcal{O}_{Y, C}$ are Cohen-Macaulay. Moreover, suppose that $j(X, Y ; C) \leqq$ length $\mathcal{O}_{X \cap Y, C}$. Then we have 


$$
j(X, Y ; C)=k(X, Y ; C)=\text { length } \mathcal{O}_{X \cap Y, C} .
$$

Proof. Since $\mathcal{O}_{X, C}$ and $\mathcal{O}_{Y, C}$ are Cohen-Macaulay we get $k(X, Y ; C)=$ length $\mathcal{O}_{X \cap Y, C}$. Therefore we obtain from Corollary 3.19 of [20]:

$$
j(X, Y ; C) \leqq \text { length } \mathcal{O}_{X \cap Y ; C}=k(X, Y ; C) \leqq j(X, Y ; C) .
$$

This shows our lemma, q.e.d.

Theorem 5. With the same notations as in Theorem 1, we assume for all $C \in \mathcal{C}_{h}(X, Y)$ that $\mathcal{O}_{X, C}$ and $\mathcal{O}_{Y, C}$ are Cohen-Macaulay, and $j(X, Y ; \mathbb{C}) \leqq$ length $\mathcal{O}_{X \cap Y, C}$. Then the following conditions are equivalent:

(i) $e \leqq r_{i j}$

(ii) $e=r_{i j}$

(iii) $\operatorname{deg} X \cdot \operatorname{deg} Y \leqq \sum_{C \in \mathcal{C}_{h}} j(X, Y ; C) \operatorname{deg} C$

(iv) $\operatorname{deg} X \cdot \operatorname{deg} Y=\sum_{C \in \mathcal{C}_{h}} j(X, Y ; C) \operatorname{deg} C$

Proof. (i) $\Rightarrow$ (iii) follows from Theorem 1. (iv) $\Rightarrow$ (ii): Our lemma 4 provides $j(X, Y ; C)=k(X, Y ; C)$. Therefore the theorem of [22] yields our condition (ii). By Lemma 1 we have (iii) $\Rightarrow$ (iv), q.e.d.

Corollary 5.1. With the same notations as in Corollary 4.1, suppose for all $C \in \mathcal{C}_{h}(X, Y)$ that $\mathcal{O}_{X, C}$ and $\mathcal{O}_{Y, C}$ are Cohen-Macaulay, and $j(X, Y: C) \leqq$ Length $\mathcal{O}_{X \cap Y, C}$. Then $e=r$ if and only if

$$
\operatorname{deg} X \cdot \operatorname{deg} Y=\sum_{C \in \mathcal{C}_{h}} j(X, Y ; C) \operatorname{deg} C \text {. }
$$

In case $r=0$ our approach now yields the following result.

Corollary 5.2. Let $X, Y$ be irreducible and reduced subschemes of $\mathbb{P}^{n}$ such that $X \cup Y$ is not lying in a hyperplane. We assume for all irreducible components $C$ of $X \cap Y$ that $\mathcal{O}_{X, C}$ and $\mathcal{O}_{Y, C}$ are Cohen-Macaulay, and $j(X, Y ; C) \leqq$ length $\mathcal{O}_{X \cap Y, C}$. Then the following conditions are equivalent:

(i) $e=0$

(ii) $\operatorname{deg} X \cdot \operatorname{deg} Y=\sum_{C \in \mathcal{C}_{\mathrm{irr}}} j(X, Y ; C) \operatorname{deg} C$

(iii) $\operatorname{deg} X \cdot \operatorname{deg} Y=\sum_{C \in \mathcal{C}_{h}} j(X, Y ; C) \operatorname{deg} C$

(iv) $\operatorname{deg} X \cdot \operatorname{deg} Y=\sum_{C \in \mathcal{C}_{\mathrm{irr}}} k(X, Y ; C) \operatorname{deg} C$

(v) $\operatorname{deg} X \cdot \operatorname{deg} Y=\sum_{C \in \mathcal{C}_{h}} k(X, Y ; C) \operatorname{deg} C$

Proof. Lemma 4 shows that $j(X, Y ; C)=k(X, Y ; C)$. Therefore Lemma 1 
and 2 provide Corollary 5.2.

L. O'Carroll's idea in [12] of bounding the intersection multiplicity $j(X, Y ; C)$ is indeed a key assumption in some of our above results. We therefore want to investigate such bounds.

Theorem 6. Let $X, Y$ be pure dimensional projective subschemes of $\boldsymbol{P}^{n}$, say $X=\bigcup_{i=1}^{r} X_{i}$ and $Y=\bigcup_{j=1}^{s} Y_{j}$ where $X_{i}$ and $Y_{j}$ are the irreducible components of $X$ and $Y$, resp. Let $C$ be an element of $\mathcal{C}(X, Y)$. We set $c:=\operatorname{dim} X \cap Y-\operatorname{dim} C \geqq 0$. Consider all integers $i, 1 \leqq i \leqq r$, and $j, 1 \leqq j \leqq s$, such that $C \leqq X_{i}$ and $C \leqq Y_{j}$, say $i=1, \cdots, r^{\prime} \leqq r$ and $j=1, \cdots, s^{\prime} \leqq s$. Let $t \geqq 0$ be the integer such that $\cup_{i=1}^{r^{\prime}} X_{i} \cup \cup_{j=1}^{s^{\prime}} Y_{j}$ is contained in an $(n-t)$-plane but not lying in an $(n-t-1)$ plane. We assume that $t \geqq e(X, Y)-c$. Then we get

$$
j(X, Y ; C)=k(X, Y ; C)
$$

Moreover, if $C$ is an irreducible component of $X \cap Y$ then we obtain:

$$
j(X, Y ; C) \leqq l\left(\mathcal{O}_{X \cap Y ; c}\right),
$$

and we have equality if and only if the local rings $\mathcal{O}_{X, C}$ and $\mathcal{O}_{Y, C}$ are CohenMacaulay.

Proof. Let $I(C)$ be the defining prime ideal of $C$. Using our notations of $\S 1$ we see that $I(C)+\mathfrak{c}$ is a prime ideal belonging to $\mathfrak{a}_{c}+l_{n-e+c}$. Let $Q \supseteqq$ $a_{c}+l_{n-e+c}$ be the primary ideal belonging to $I(C)+c$. We now consider the intersection algorithm in the join construction as developed in $\S 1$. Moreover, take this algorithm with respect to the localization at $I(C)+\mathrm{c}$. Then we get

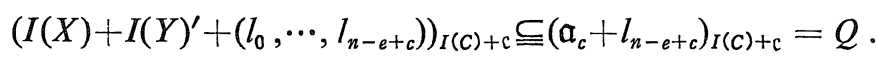

Since $t \geq e-c$ we obtain that

$$
\mathfrak{c} \subseteq\left(I(X)+I(Y)^{\prime}+\left(l_{0}, \cdots, l_{n-e-c}\right)\right)_{I(c)+c} \subseteq Q
$$

This shows that $j(X, Y ; C)=k(X, Y ; C)$. If $C$ is an irreducible component then we have length $\left(\mathcal{O}_{X \cap Y, C}\right) \geqq$ length $Q$. Since $c \subseteq Q$ we get from Corollary 3.19 of [20] and lemma 3 of [1] that $j(X, Y ; C)=$ length $\left(\mathcal{O}_{X \cap Y, C}\right)$ if and only if $\mathcal{O}_{X, C}$ and $\mathcal{O}_{Y, C}$ are Cohen-Macaulay. This completes the proof of theorem 6 , q.e.d.

Finally, we want to prove a result in terms of length-multiplicity. We first recall that the degree of a closed subscheme $W \subset \mathbb{P}^{n}$ is given by 


$$
\operatorname{deg} W=\sum_{C} \operatorname{length}\left(\mathcal{O}_{W, C}\right) \cdot \operatorname{deg} C
$$

where the sum is taken over all irreducible components $C$ with $\operatorname{dim} C=\operatorname{dim} W$. Letting $C$ run over all irreducible components we set

$$
\widetilde{\operatorname{deg}} W:=\sum_{C} \operatorname{length}\left(\mathcal{O}_{W, C}\right) \cdot \operatorname{deg} C \text {. }
$$

Theorem 7. Let $X, Y$ be reduced and pure dimensional subschemes of $\mathbb{P}_{k}^{n}$ with defining ideal $I(X)$ and $I(Y)$ in $k\left[x_{0}, \cdots, x_{n}\right]=: R$. We assume that

(i) $X$ and $Y$ are locally Cohen-Macaulay

(ii) depth $R / I(X) \geqq 2$ and depth $R / I(Y) \geqq 2$

(iii) $\operatorname{dim} X+\operatorname{dim} Y-n \geqq 0$

(iv) $X \cup Y$ is not lying in a hyperplane

Then we have

$$
\operatorname{deg} X \cdot \operatorname{deg} Y \geqq \widetilde{\operatorname{deg}} X \cap Y+e .
$$

Proof. The assumptions (i), (ii) and (iii) do provide that $X$ and $Y$ are connected in dimension $\operatorname{dim} X-1$ and $\operatorname{dim} Y-1$, resp. For example, this follows from Theorem 5 of [16]. Let $C$ be an irreducible component of $X \cap Y$. Then condition (i) and (iii) show that

$$
k(X, Y ; C)=\text { length } \mathcal{O}_{X \cap Y, C} .
$$

Therefore Lemma 2 of $\S 1$ gives Theorem 7. q.e.d.

The proof of this theorem gives the following

Corollmary. Let $X$ and $Y$ be reduced and arithmetically Cohen-Macaulay subschemes of dimension $\geqq 1$ in $\mathbb{P}_{k}^{n}$. If $X \cup Y$ is not lying in a hyperplane then

$$
\operatorname{deg} X \cdot \operatorname{deg} Y \geqq \widetilde{\operatorname{deg}} X \cap Y+e \text {. }
$$

Question. Is this corollary true if $X$ or $Y$ is not reduced?

\section{§4. Examples and Problems}

We discuss in conclusion some examples and open questions. In connection with our remark after Lemma 3 of $\S 1$ we want to study our first example.

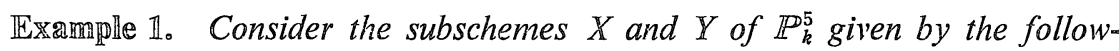
ing homogeneous ideals in $k\left[X_{0}, X_{1}, \cdots, X_{5}\right]$ :

$$
\begin{aligned}
& I(X)=\left(X_{1}^{2}, X_{2}^{2}, X_{1} X_{2}, X_{1} X_{4}-X_{2} X_{3}, X_{1} X_{5}-X_{2} X_{4}, X_{3} X_{5}-X_{4}^{2}\right), \\
& I(Y)=\left(X_{3}, X_{4}, X_{5}\right)
\end{aligned}
$$


Claim. $X$ and $Y$ are irreducible, and $X \cap Y$ has precisely one irreducible intersection component, say $C$, with defining ideal $I(C)=\left(X_{1}, X_{2}, X_{3}, X_{4}, X_{5}\right)$. Moreover, we get

$$
k(X, Y ; C)>\text { length } \mathcal{O}_{X, c_{1}} \text { length } \mathcal{O}_{Y, C_{2}} \cdot k\left(X_{\text {red }}, Y_{\text {red }} ; C\right),
$$

where $C_{1}$ is defined by $I\left(C_{1}\right)=\left(X_{1}, X_{2}, X_{3} X_{5}-X_{4}^{2}\right)$ and $C_{2}$ is given by $I\left(C_{2}\right)=$ $\left(X_{3}, X_{4}, X_{5}\right)$. We also note that $(*)$ remains true by replacing the multiplicity $k$ by the length-multiplicity.

Proof. First we show that $X$ is an irreducible and arithmetically CohenMacaulay scheme. Let $F$ be the Veronese surface in $\mathbb{P}^{5}$ given parametrically by

$$
\left\{t_{0}^{2}, t_{0} t_{1}, t_{0} t_{2}, t_{1}^{2}, t_{1} t_{2}, t_{2}^{2}\right\}
$$

Then $F$ is given by the following prime ideal

$I(F)=\left(X_{0} X_{3}-X_{1}^{2}, X_{0} X_{5}-X_{2}^{2}, X_{3} X_{5}-X_{4}^{2}, X_{0} X_{4}-X_{1} X_{2}, X_{1} X_{4}-X_{2} X_{3}, X_{1} X_{5}-X_{2} X_{4}\right)$

The defining equations of $X$ are given by $\left(I(F), X_{0}\right)$ modulo $X_{0}$. Therefore $X$ is arithmetically Cohen-Macaulay and degree $X=4$ since $F$ has these properties. Since $X_{\text {red }}$ is given by the prime $I\left(X_{\text {red }}\right)=\left(X_{1}, X_{2}, X_{3} X_{5}-X_{4}^{2}\right)$ we see that $X$ is indeed irreducible. Since $X, X_{\text {red }}$ and $Y$ are arithmetically Cohen-Macaulay we get

$$
k\left(X_{\mathrm{red}}, Y_{\mathrm{red}} ; C\right)=l\left(X_{\mathrm{red}}, Y_{\mathrm{red}} ; C\right)=1,
$$

$k(X, Y ; C)=l(X, Y ; C)=3$. Moreover, we have length $\mathcal{O}_{X, c_{1}}=2$ since $I\left(C_{1}\right)=$ $I\left(X_{\text {red }}\right)$. These properties yield our claim, q.e.d.

We will study again an example of the intersection of 3 surfaces in $\mathbb{P}^{3}$. We will obtain two extra (non-isolated) intersection points counted with multiplicities.

Example 2. Let $X, Y$ be subschemes of $\mathbb{P}_{k}^{3}$ with the following ideals in $k\left[X_{0}, \cdots, X_{3}\right]$ :

$$
I(X)=\left(X_{0}, X_{1} X_{2}\right) \text { and } I(Y)=\left(X_{1} X_{3}\right) .
$$

Then $X \cap Y$ is given by the following primary decomposition:

$$
I(X)+I(Y)=\left(X_{0}, X_{1}\right) \cap\left(X_{0}, X_{2}, X_{3}\right) .
$$

Then Theorem 3 show's that our collection $C(X, Y)$ contains, of course, the line $X_{0}=X_{1}=0$ and the point $X_{0}=X_{2}=X_{3}=0$, and moreover, two extra non-isolated intersection points: $X_{0}=X_{1}=X_{2}=0$ and $X_{0}=X_{1}=X_{3}=0$. All elements of 
$\mathcal{C}(X, Y)$ have multiplicity one.

The third example shows again that non-isolated intersection points of 3 surfaces in $\mathbb{P}^{3}$ have not always multiplicities in our intersection theory. Moreover, this example establishes also our theorems $1,2,4$ and our remark below.

Example 3. Let $X$ and $Y$ be subschemes of $\mathbb{P}_{k}^{3}$ with the following ideals in $k\left[X_{0}, \cdots, X_{3}\right]$ :

Then

$$
I(X)=\left(X_{2}^{2}, X_{1} X_{2}-X_{3}^{2}\right) \text { and } I(Y)=\left(X_{3}\right) \text {, resp. }
$$

$$
I(X)+I(Y)=\left(X_{2}^{2}, X_{3}, X_{1} X_{2}\right)=\left(X_{2}, X_{3}\right) \cap\left(X_{1}, X_{2}^{2}, X_{3}\right) \text {. }
$$

For example, Theorem 1 and Lemma 1 show that $\mathcal{C}(X, Y)=\mathcal{C}_{h}(X, Y)$. Therefore the line $X_{2}=X_{3}=0$ has multiplicity 4 , and the embedded point $X_{1}=X_{2}=X_{3}$ $=0$ has thus no multiplicity.

Finally, we want to state two problems.

Problem 1 (see also [20]). Let $X$ and $Y$ be reduced and irreducible subschemes of $\mathbb{P}_{k}^{n}$ such that the union $X \cup Y$ is not lying in a hyperplane. Describe contributions of non-isolated components of $X \cap Y$ to the intersection theory.

Remark. If $X$ or $Y$ is not reduced then our paper does provide some results on contributions of non-isolated components to intersection theory. A reason for this is the following fact:

Let $C$ be an irreducible component of $X \cap Y$. It is well-known that

$$
\operatorname{dim} C \geqq \operatorname{dim} X+\operatorname{dim} Y-n .
$$

But this lower bound is not sharp in general (see the first proof of this bound given in [23]). Indeed, we have the following correction term:

Let $\mathfrak{a}$ and $\mathfrak{b}$ homogeneous ideals of the polynomial ring $k\left[X_{0}, \cdots, X_{n}\right]$. Let $\mathfrak{p}$ be a minimal prime ideal belonging to $\mathfrak{a}+\mathfrak{b}$. Then we get for the (homogeneous) dimension:

$$
\operatorname{dim} \mathfrak{p} \geqq \operatorname{dim} \mathfrak{a}+\operatorname{dim} \mathfrak{b}-n \dot{+} \operatorname{dim}_{k}[\mathbb{R a d} \mathfrak{a} \cap \operatorname{Rad} \mathfrak{b}]_{1},
$$

where $[\operatorname{Rad} \mathfrak{a} \cap \operatorname{Rad} \mathfrak{b}]_{1}$ is the vector space of all forms of dgeree 1 contained in $\operatorname{Rad} \mathfrak{a} \cap \operatorname{Rad} \mathfrak{b}$, and $\operatorname{Rad}(\cdots)$ is the radical of the ideal $(\cdots)$.

In this sense we can replace the condition $e=0$ for a proper intersection by the assumption

$$
e=\operatorname{dim}_{k}[\operatorname{Rad} a \cap \operatorname{Rad} \mathfrak{b}]_{1} \text { (see, e.g., our theorems } 1,2,4,5 \text { ). }
$$

Problem 2 (see also [17]). Give an arithmetic version of Bézout's theo- 
rem. However, Christophe Soulé informed me during the international congress of mathematicians in Kyoto that he has now an approach for solving this problem. Therefore we want to state an extended problem:

Describe a refined Bézout's theorem (see our lemma 1 of $\S 1$ ) in algebraic and arthmetic geometry.

\section{Acknowledgement}

The author thanks the Research Institute for Mathematical Sciences in Kyoto for hospitality and financial support during the preparation of this paper.

\section{References}

[1] Achilles, R., Huneke, C. and Vogel, W., A criterion for intersection multiplicity one, Nagoya Math. J., 100 (1985), 49-63.

[2] Flenner, H. and Vogel, W., Connectivity and its applications to improper intersection in $P^{n}$, Mathematica Gottingensis, Heft 53, Göttingen 1988.

[ 3 ] Flenner, H., Gastel, L. van and Vogel, W., Joins and intersections, to appear in Math. Ann.

[ 4 ] Fulton, W., Intersection theory, Berlin, Heidelberg, New York, Tokyo, Springer 1984.

[5] Gaeta, F., Associate forms, joins, multiplicities and an intrinsic elimination theory, In: Topics in Algebra, Banach Center Publications, 26, Part 2, 71-108, PWN Polish Scientific Publishers, Warsaw 1990.

[6] Gastel, L. van, A geometric approach to Vogel's intersection theory, Preprint 427, University of Utrecht, The Netherlands, 1986/87.

[7] - Excess intersections and a correspondence principle, Invent. Math., 103 (1991), 197-221.

[8] - Excess intersections in projective space, In: Topics in Algebra, Banach Center Publications, Volume 26, Part 2, 109-124, PWN Polish Scientific Publishers, Warsaw 1990.

[9] Gröbner, W., Über die Eliminationstheorie, Monatsh. Math., 54 (1950), 71-78.

[10] — Über den Multiplizitätsbegriff in der algebraischen Geometrie, Math. Nachr., 4 (1950/51), 193-201.

[11] Kanemitsu, M. and Yoshida, K., On embedded primary components, Osaka J. Math., 26 (1989), 665-670.

[12] O'Carroll, L., On bounding the Stückrad-Vogel multiplicity, Proc. Edinburgh Math. Soc. (2), 34 (1991), 251-257.

[13] Perron, O., Studien über den Vielfachheitsbegriff und den Bezoutschen Satz, Math. Zeitschr., 49 (1944), 654-680.

[14] Pruschke, T., Notes on questions of W. Vogel concerning the converse to Bézout's theorem, submitted.

[15] Renschuch, B. und Vogel, W., Perfektheit und die Umkehrung des Bezoutschen Satzes, Math. Nachr., 148 (1990), 313-323.

[16] Roloff, H. and Stückrad, J., Bemerkungen über Zusammenhangseigenschaften und mengentheoretische Darstellung projektiver algebraischer Mannigfaltigkeiten, Beitr. Algebra Geom., 8 (1979), 125-131.

[17] Soulé, C., Geometrie d'Arakelov et theorie des nombres transcendants, Preprint, In- 
stitut des Hautes Etudes Scientifiques, M/90/2. Bures sur Yvette, 1990.

[18] Stückrad, J. and Vogel, W., An algebraic approach to the intersection theory, In: The curves seminar at Queen's Vol. II, 1-32. Queen's papers in pure and applied mathematics, No. 61. Kingston, Ontario, Canada, 1982.

[19] Stückrad, J. and Vogel, W., An Euler-Poincaré characteristic for improper intersections, Math. Ann., 274 (1986), 257-271.

[20] Vogel, W., Lectures on results on Bezout's theorem, Tata lecture notes, Bombay, No. 74. Berlin, Heidelberg, New York, Tokyo, Springer 1984.

[21] — A converse of Bézout's theorem and applications, Short communication at International Congress of Mathematicians, Kyoto, Japan, August 21-29, 1990.

[22] — A converse of Bézout's theorem, to appear in Ann. Univ. Ferrara Sez VII (N.S.).

[23] Waerden, B.L. van der, Zur algebraischen Geomerrie. XII. Ein Satz über Korrespondenzen und die Dimension einer Schnittmannigfaltigkeit, Math. Ann., $\mathbb{1} \mathbb{1} 5$ (1938), 330-332.

[24] — Review, Zentralblatt für Mathematik, 25 (1942), 211-212.

[25] Yokura, S., Polar classes and Segre classes for singular projective varieties, Trans. Amer. Math. Soc., 298 (1986), 169-191.

[26] - A formula for Segre classes of singular projective varieties, Pacific $J$. Math., 146 (1990), 385-394.

Note added in Proof: See also the following paper: Flenner, H. and Vogel, W., Improper intersections and a converse to Bezout's theorem, to appear in J. Algebra. 